\title{
1. QUANTUM COMPUTING
}

Quantencomputing bietet eine völlig neuartige Möglichkeit, komplexe Berechnungen sehr viel schneller und oftmals überhaupt erst auf praktische Weise durchzuführen.

Diese bahnbrechende Entwicklungen, die in den letzten Jahren große Sprünge in der Praxis erlebt hat, geht auf zwei wissenschaftliche Revolutionen des frühen 20. Jahrhunderts zurück. Die erste Revolution wurde um 1930 durch die radikal neuartige Theorie der Quantenmechanik ausgelöst, die unsere Auffassung von Realität drastisch verändert hat. Die zweite wissenschaftliche Revolution erfolgte in den 1940er Jahren, indem die Grundlagen für den Bau erster programmierbarer Computer gelegt wurden, welche die Basis aller Rechentecnik ist, wie wir sie heute von Smartphones bis Großrechner kennen. In den letzten beiden Jahrzehten wurden diese beiden Wissenschaften zusammen geführt und es entstand der interdisziplinäre Zweig des Quantencomputings.

Quantencomputer sind Rechenmaschinen, welche die Effekte der Quantenmechanik verwenden. Dies beinhaltet die Fähigkeit mehrere Zustände gleichzeitig zu besitzen (Superposition), mit einer Operation viele Zustände gleichzeitig zu verändern (Verschränkung) sowie unwahrscheinliche Lösungen zielstrebig zu erreichen (Tunneling). Neben universellen Quantencomputern spielen sogenannte Quantenannealer eine immens wichtige Rolle, da sie besonders dafür konzipiert sind, Optimierungsprobleme zu lösen.

In dieser Ausgabe erwarten Sie Artikel rund um das Thema Quantencomputing. Die Themen fokussieren technische Hintergründe, mögliche Anwendungsfälle sowie visionäre Gedanken.

\section{INHALT}

\section{FACHBEITRÄGE}

Jan-Rainer Lahmann | Ein spielerischer Einstieg in Quantum Computing

PlanQK - Quantum Computing Meets Artificial Intelligence How to make an ambitious idea reality

Roman Uminski | Wie Quantenalgorithmen die Informationstechnologie revolutionieren

Roman Uminski | Welche Auswirkungen hat das Quantum Computing auf die Informationssicherheit

\section{INTERVIEWS}

Faisal Shah Khan | Very Digital Person

Prof. Dr. Gerhard Kirchmair | The Physics Behind the Quantum Computer

Prof. Rudolf Gross | "Munich Takes the Lead In Quantum Science."

\section{BLOGBEITRÄGE}

Annie Bailey | Künstliche Intelligenz und Quantencomputing Eine natürliche Trendwende

Roman Uminski | Kann Quantum Comouting den Klimawandel stoppen 